\title{
First Extraction of Transversity from a Global Analysis of Electron-Proton and Proton-Proton Data
}

\author{
Marco Radici ${ }^{1, *}$ and Alessandro Bacchetta ${ }^{2,1, \dagger}$ \\ ${ }^{1}$ INFN Sezione di Pavia, Via Bassi 6, I-27100 Pavia, Italy \\ ${ }^{2}$ Dipartimento di Fisica, Università di Pavia, via Bassi 6, I-27100 Pavia, Italy
}

(Received 26 February 2018; revised manuscript received 5 April 2018; published 10 May 2018)

\begin{abstract}
We present the first extraction of the transversity distribution in the framework of collinear factorization based on the global analysis of pion-pair production in deep-inelastic scattering and in proton-proton collisions with a transversely polarized proton. The extraction relies on the knowledge of dihadron fragmentation functions, which are taken from the analysis of electron-positron annihilation data. For the first time, the transversity is extracted from a global analysis similar to what is usually done for the spinaveraged and helicity distributions. The knowledge of transversity is important for, among other things, detecting possible signals of new physics in high-precision low-energy experiments.
\end{abstract}

DOI: 10.1103/PhysRevLett.120.192001

Parton distribution functions (PDFs) describe the internal structure of hadrons in terms of number densities of confined quarks and gluons. If parton transverse momenta are integrated over, the complete spin structure of the nucleon at leading twist is described in terms of three PDFs: the spin-averaged $\operatorname{PDF} f_{1}$, the helicity $\operatorname{PDF} g_{1}$, and the transversity PDF $h_{1}$. The knowledge of PDFs is crucial for the interpretation of high-energy experiments involving hadrons and for detecting signals of new physics beyond the standard model (BSM). Transversity has recently received increasing attention because of the importance of a precise determination of its integral, the so-called tensor charge $\delta q$ (for a flavor $q$ ) [1]. In neutron $\beta$ decays, BSM effects can arise from the interference between standard model operators and a new possible tensor operator whose coupling involves $\delta q$ [2]. Similarly, $\delta q$ enters the expression of the fermionic electric dipole moment that could constrain new possible $C P$-violating couplings in some BSM theories [3,4]. In this Letter, we present an extraction of transversity at leading order (LO) in the strong coupling constant $\alpha_{s}$. For the first time, we perform a global analysis of data on deep-inelastic scattering (DIS) and proton-proton collisions; i.e., for the first time, we make a global analysis similar to what is usually done for the spin-averaged and the helicity distributions.

The PDFs $f_{1}$ and $g_{1}$ are nowadays fairly well determined (see, e.g., Refs. $[5,6]$ and references therein). On the contrary, we have a limited knowledge on $h_{1}$. Transversity

Published by the American Physical Society under the terms of the Creative Commons Attribution 4.0 International license. Further distribution of this work must maintain attribution to the author(s) and the published article's title, journal citation, and DOI. Funded by SCOAP . describes the correlation between the transverse polarization of the nucleon and the transverse polarization of its constituent partons. The chiral-odd nature of $h_{1}$ makes it hard to extract it from experimental data because, in the cross section, transversity must always be paired to another chiral-odd object [7]. Transversity was extracted for the first time from data on single-particle semi-inclusive DIS (SIDIS) [8] where the cross section contains an azimuthal modulation proportional to the convolution $h_{1} \otimes H_{1}^{\perp}$ involving the chiral-odd Collins fragmentation function $H_{1}^{\perp}$ [9]. However, the convolution $h_{1} \otimes H_{1}^{\perp}$ involves the transverse momenta of quarks. Therefore, the evolution of the various partonic functions among different experimental energies must be described in the framework of transversemomentum-dependent factorization, which depends also on nonperturbative parameters [10]. More importantly, this analysis of the so-called Collins effect cannot be extended to hadronic collisions because of factorization breaking contributions [11].

An alternative method to access the transversity PDF requires only standard collinear factorization, thus avoiding the above complications and limitations. It considers the semi-inclusive production of two hadrons with small invariant mass [12] and is based on the correlation between the transverse polarization of the quark fragmenting into the two hadrons and their transverse relative momentum [13]. In this case, the dihadron SIDIS cross section (once integrated over partonic transverse momenta) contains a specific modulation in the azimuthal orientation of the plane containing the momenta of the two hadrons. The coefficient of this modulation is the simple product $h_{1} H_{1}^{\varangle}$, where $H_{1}^{\varangle}$ is a chiral-odd dihadron fragmentation function (DiFF) quantifying the above correlation [14-16]. The function $H_{1}^{\varangle}$ can be independently determined by looking at correlations between the azimuthal orientations of two 
hadron pairs in back-to-back jets in $e^{+} e^{-}$annihilation [17-19]. The advantage of this method with respect to the Collins effect is that collinear factorization makes it possible to isolate the same combination $h_{1} H_{1}^{\varangle}$ also in proton-proton collisions [20], giving rise to an azimuthally asymmetric distribution of the final hadron pair when one of the two initial protons is transversely polarized [21].

Experimental data for the SIDIS asymmetry in the azimuthal distribution of final $\left(\pi^{+} \pi^{-}\right)$pairs were first collected by the HERMES Collaboration for a transversely polarized proton target [22], and by the COMPASS Collaboration for polarized protons and deuterons [23-25]. The azimuthal asymmetry in the distribution of back-to-back $\left(\pi^{+} \pi^{-}\right)$pairs in $e^{+} e^{-}$annihilation was measured by the Belle Collaboration [26], opening the way to the first parametrization of $H_{1}^{\varangle}$ for the up and down quarks [19]. This result was used in combination with the SIDIS data to extract the valence components of $h_{1}$ [27-29]. Recently, the STAR Collaboration released the first results for the relevant asymmetry in the azimuthal distribution of $\left(\pi^{+} \pi^{-}\right)$pairs produced in proton-proton collisions with a transversely polarized proton [30]. Here, we present for the first time the extraction of the transversity $\mathrm{PDF} h_{1}$ from a global fit of all these data.

For the SIDIS process $e[k]+A^{\uparrow}[P] \rightarrow e^{\prime}\left[k^{\prime}\right]+\left(\pi^{+} \pi^{-}\right)\left[P_{h}\right]+X$, where an electron with 4-momentum $k$ scatters off a transversely polarized proton $(A=p)$ or deuteron $(A=d)$ with 4-momentum $P$ at the hard scale $Q^{2}=-q^{2}=-\left(k-k^{\prime}\right)^{2} \geq 0$ producing a $\left(\pi^{+} \pi^{-}\right)$pair with total 4-momentum $P_{h}$ and relative 4-momentum $R$ plus any number of undetected hadrons $(X)$, the relevant asymmetry at leading twist (usually denoted as $A_{U T}^{\sin \left(\phi_{R}+\phi_{S}\right) \sin \theta}$ [27-29]) can be written as

$$
A_{\mathrm{DIS}}\left(x, Q^{2}\right)=-C_{y} \frac{\sum_{q} e_{q}^{2} h_{1}^{q}\left(x, Q^{2}\right) n_{q}^{\uparrow}\left(Q^{2}\right)}{\sum_{q} e_{q}^{2} f_{1}^{q}\left(x, Q^{2}\right) n_{q}\left(Q^{2}\right)},
$$

where $x=Q^{2} /(2 P \cdot q)$ is the fractional momentum of the initial quark, $C_{y}$ is a coefficient depending on the average value of the fractional beam energy loss $y=P \cdot q /(P \cdot k), e_{q}$ is the fractional electric charge of a quark $q$, and

$$
\begin{aligned}
& n_{q}\left(Q^{2}\right)=\int d z \int d M_{h} D_{1}^{q}\left(z, M_{h}, Q^{2}\right), \\
& n_{q}^{\uparrow}\left(Q^{2}\right)=\int d z \int d M_{h} \frac{|\boldsymbol{R}|}{M_{h}} H_{1}^{\varangle q}\left(z, M_{h}, Q^{2}\right),
\end{aligned}
$$

where $z=P \cdot P_{h} /(P \cdot q)=z_{\pi^{+}}+z_{\pi^{-}}$is the fractional energy carried by the $\left(\pi^{+} \pi^{-}\right)$pair, $M_{h}$ is its invariant mass $\left(M_{h}^{2}=P_{h}^{2} \ll Q^{2}\right)$, and the DiFFs $D_{1}^{q}$ and $H_{1}^{\varangle q}$ describe the fragmentation into the $\left(\pi^{+} \pi^{-}\right)$pair of an unpolarized or transversely polarized quark $q$, respectively. Data were collected in bins of $x, z$, and $M_{h}$ for $\left(\pi^{+} \pi^{-}\right)$pairs and for final unidentified $\left(h^{+} h^{-}\right)$pairs [23,24]. Since our goal is to extract transversity from data for the inclusive $\left(\pi^{+} \pi^{-}\right)$production, here we consider the data set for identified $\left(\pi^{+} \pi^{-}\right)$pairs with only the bins in $x[22,25]$, whose average value spans the range $0.0065 \leq\langle x\rangle \leq 0.133$ corresponding to the average scale range $1.232 \leq\left\langle Q^{2}\right\rangle \leq 31.5 \mathrm{GeV}^{2}$.

The unknown factors $n_{q}, n_{q}^{\uparrow}$ of Eq. (2) can be inferred from the process $e^{+}[\bar{k}]+e^{-}[k] \rightarrow\left(\pi^{+} \pi^{-}\right)\left[P_{h}\right]+\left(\pi^{+} \pi^{-}\right)\left[\bar{P}_{h}\right]+X$ at the hard scale $Q^{2}=(k+\bar{k})^{2} \geq 0$, where the two $\left(\pi^{+} \pi^{-}\right)$ pairs are emitted in opposite hemispheres (ensured by the condition $\left.P_{h} \cdot \bar{P}_{h} \approx Q^{2}\right)$. By summing the $\left(\pi^{+} \pi^{-}\right)$pairs of one hemisphere, the so-called Artru-Collins asymmetry (usually denoted as $A^{\cos \left(\phi_{R}+\phi_{\bar{R}}\right)}$ [17-19,31,32] or $a_{12 R}$ in the Belle publication [26]) is given by

$$
\begin{aligned}
A_{e^{+} e^{-}}\left(z, M_{h}, Q^{2}\right)= & -C_{\theta_{2}} \frac{|\boldsymbol{R}|}{M_{h}} \\
& \times \frac{\sum_{q} e_{q}^{2} H_{1}^{\varangle q}\left(z, M_{h}, Q^{2}\right) n_{q}^{\uparrow}\left(Q^{2}\right)}{\sum_{q} e_{q}^{2} D_{1}^{q}\left(z, M_{h}, Q^{2}\right) n_{q}\left(Q^{2}\right)},
\end{aligned}
$$

where $C_{\theta_{2}}$ is a coefficient depending on the average value of the angle $\theta_{2}$ between the $\overline{\boldsymbol{k}}$ and $\boldsymbol{P}_{h}$ directions. The $D_{1}^{q}$ is parametrized to reproduce the two-pion yield of the PYTHIA event generator tuned to the Belle kinematics [19]. Data span the range $0.2 \leq z \leq 1$ and $0.3 \leq M_{h} \leq 1.2 \mathrm{GeV}$ at $Q=10 \mathrm{GeV}$. (When this Letter was being finalized, the Belle Collaboration officially published the first data for the differential dihadron multiplicities [33]. We will use these data in a future work to parametrize the unpolarized DiFF $D_{1}$ directly from experiment.)

The same elementary mechanism $h_{1} H_{1}^{\varangle}$ active in SIDIS generates an azimuthal asymmetry also in the $p\left[P_{A}\right]+$ $p^{\uparrow}\left[P_{B}\right] \rightarrow\left(\pi^{+} \pi^{-}\right)\left[P_{h}\right]+X$ process [20], where a proton with 4-momentum $P_{A}$ collides with a transversely polarized proton with 4-momentum $P_{B}$. After integrating over the partonic transverse momenta, the total 4-momentum $P_{h}$ of the $\left(\pi^{+} \pi^{-}\right)$pair has no transverse component with respect to the fragmenting quark momentum, but it can have the transverse component $\boldsymbol{P}_{h T}$ with respect to $P_{A}$. The $\boldsymbol{P}_{h T}^{2}$ represents the hard scale of the process $\left(\boldsymbol{P}_{h T}^{2} \gg M_{h}^{2}=P_{h}^{2}\right)$. If we identify the reaction plane by $\left(\boldsymbol{P}_{A}, \boldsymbol{P}_{h}\right)$, the relevant asymmetry in the azimuthal distribution of $\left(\pi^{+} \pi^{-}\right)$pairs with respect to the reaction plane (usually denoted as $A_{U T}$ $[21,30])$ is given at leading twist by

$$
A_{p p}\left(\eta,\left|\boldsymbol{P}_{h T}\right|, M_{h}\right)=\frac{\pi}{4} \frac{|\boldsymbol{R}|}{M_{h}} \frac{H\left(\eta,\left|\boldsymbol{P}_{h T}\right|, M_{h}\right)}{D\left(\eta,\left|\boldsymbol{P}_{h T}\right|, M_{h}\right)},
$$

where $\eta$ is the pseudorapidity of the hadron pair with respect to the beam $\boldsymbol{P}_{A}$, and

$$
\begin{aligned}
H\left(\eta,\left|\boldsymbol{P}_{h T}\right|, M_{h}\right)= & \sum_{a, b, c, d} \int \frac{d x_{a} d x_{b}}{z_{h}} f_{1}^{a}\left(x_{a}\right) h_{1}^{b}\left(x_{b}\right) \\
& \times \frac{d \Delta \sigma_{a b^{\uparrow} \rightarrow c^{\uparrow} d}}{d \hat{t}} H_{1}^{\varangle c}\left(z_{h}, M_{h}\right),
\end{aligned}
$$




$$
\begin{aligned}
D\left(\eta,\left|\boldsymbol{P}_{h T}\right|, M_{h}\right)= & \sum_{a, b, c, d} \int \frac{d x_{a} d x_{b}}{z_{h}} f_{1}^{a}\left(x_{a}\right) f_{1}^{b}\left(x_{b}\right) \\
& \times \frac{d \sigma_{a b \rightarrow c d}}{d \hat{t}} D_{1}^{c}\left(z_{h}, M_{h}\right) .
\end{aligned}
$$

The dependence upon the hard scale $\boldsymbol{P}_{h T}^{2}$ is understood in all PDFs and DiFFs. The elementary annihilation of partons $a$ and $b$ (carrying fractional momenta $x_{a}$ and $x_{b}$, respectively) into the partons $c$ and $d$ is described by the cross section $d \sigma$, while $d \Delta \sigma$ refers to the transfer of transverse polarization in the same mechanism [20]. Both cross sections are differential in $\hat{t}=t x_{a} / z_{h}$, where $t=\left(P_{A}-P_{B}\right)^{2}$ and $z_{h}$ is the fractional energy carried by the pion pair, which is related by momentum conservation to $\boldsymbol{P}_{h T}^{2}, \eta, x_{a}, x_{b}$, and $s=$ $\left(P_{A}+P_{B}\right)^{2}$ (the squared center-of-mass energy in the collision) [20]. Data for $A_{p p}$ were collected by the STAR Collaboration at $\sqrt{s}=200 \mathrm{GeV}[30]$ in bins of $\eta,\left|\boldsymbol{P}_{h T}\right|$, and $M_{h}$, after integrating on the complementary variables. The average values are limited to the ranges $-0.84 \leq \eta \leq 0.84$, $3 \leq\left|\boldsymbol{P}_{h T}\right| \leq 13 \mathrm{GeV}$, and $0.3 \leq M_{h} \leq 1.2 \mathrm{GeV}$, which correspond to $0.15 \lesssim\langle x\rangle \lesssim 0.3$ in SIDIS but at a larger hard scale.

In order to reduce the computational time, following Ref. [34], we rewrite the parameter-dependent part of Eq. (5) in Mellin space (see Supplemental Material [35]). In order to exploit this workaround, it is crucial that the Mellin transform of $h_{1}$ can be analytically calculated at any scale. The functional form adopted in previous fits of dihadron SIDIS data does not match this criterion [28,29]. Here, we have modified it, but kept its main features: (a) satisfying the Soffer inequality at any scale $Q^{2}$ and (b) having a high degree of flexibility with up to three nodes in $x$. Since the Soffer bound is valid for each quark and antiquark and we need to parametrize their valence combination $q_{v}=q-\bar{q}$, we constrain the transversity by taking the sum of Soffer bounds for both quarks and antiquarks [28]. At $Q_{0}^{2}=1 \mathrm{GeV}^{2}$, the general structure of the functional form is given by

$$
x h_{1}^{q_{v}}\left(x, Q_{0}^{2}\right)=F^{q}(x) F_{\mathrm{SB}}^{q}(x),
$$

where $F_{\mathrm{SB}}^{q}(x)$ is a fit to the sum of the Soffer bounds for $q$ and $\bar{q}$ at $Q_{0}^{2}[35]$ and

$$
\begin{aligned}
F^{q}(x) & =N_{F}^{q} \frac{\mathcal{F}^{q}(x)}{\max _{x}\left[\left|\mathcal{F}^{q}(x)\right|\right]}, \\
\mathcal{F}^{q}(x) & =x^{A_{q}}\left[1+B_{q} T_{1}(x)+C_{q} T_{2}(x)+D_{q} T_{3}(x)\right] .
\end{aligned}
$$

The $T_{n}(x)$ are the Chebyshev polynomials of order $n$. The $N_{F}^{q}, A_{q}, B_{q}, C_{q}$, and $D_{q}$ are fitting parameters. Simplifying assumptions on the isospin symmetry of DiFFs allow us to access only the valence components of transversity [27,36]; hence, we have in total ten free parameters. If we impose the constraint $\left|N_{F}^{q}\right| \leq 1$, then $\left|F^{q}(x)\right| \leq 1$ for all $x$, and the transversity of Eq. (7) automatically satisfies the Soffer inequality at any scale [37]. In this Letter, we keep for $f_{1}$ and $g_{1}$ the same parametrizations as in our previous fits (MSTW08 at LO for $f_{1}$ [38] and DSSV for $g_{1}$ [39]). (We checked that replacing DSSV with more recent parametrizations of $g_{1}$ (like JAM15 [40] or JAM17 [41]) does not make any relevant change to our results within the current experimental and theoretical uncertainties.) With this choice, the Soffer bound at $Q_{0}^{2}$ can be reproduced by the $F_{\mathrm{SB}}$ at $1 \%$ accuracy in the range $0.001 \leq x \leq 1$ [35]. From Eqs. (7) and (8), we deduce that $x h_{1}^{q_{v}}(x) \approx x^{A_{q}+a_{q}}$ at very small $x$. This asymptotic behavior is strongly constrained by requiring that the tensor charge

$$
\delta q\left(Q^{2}\right)=\int_{0}^{1} d x h_{1}^{q_{v}}\left(x, Q^{2}\right)
$$

is finite. We numerically evaluate the integral in the range $\left[x_{\min }, 1\right]$ where for MSTW08 $x_{\min }=10^{-6}$ [38]. In order to avoid uncontrolled extrapolation errors below $x_{\min }$, we impose the condition $A_{q}+a_{q}>1 / 3$, which also grants that $\delta q$ is evaluated at $1 \%$ accuracy. (According to Ref. [42], the more stringent condition $A_{q}+a_{q}>1$ is required to avoid a violation of the Burkardt-Cottingham sum rule by an infinite amount.)

By inserting Eq. (8) in Eq. (7) and using the function $F_{\mathrm{SB}}$ listed in [35], the resulting expression can be easily transformed in Mellin space and evolved at LO [35]. When dealing with the $A_{\mathrm{DIS}}$ of Eq. (1), the $h_{1}\left(x, Q_{0}^{2}\right)$ of Eq. (7) is evolved using the HOPPET code [43] suitably extended to include LO chiral-odd splitting functions (and, similarly, for $H_{1}^{\varangle}$ [44]).

The statistical uncertainty of the global fit is studied using the same bootstrap method as in our previous fits $[28,29]$. In the following, for a set of $M$ replicas of the data points, the statistical error is constructed by taking the central $90 \%$ of them, namely, by rejecting the largest and smallest $5 \%$ of the $M$ replicas for each experimental bin. The theoretical result is obtained by integrating the asymmetry over the bin width of the displayed variable, after integrating over the full range of the other ones. In the analysis of dihadron $e^{+} e^{-}$data, the $D_{1}^{q}$ with $q=u, d, s, c$, is parametrized from the PYTHIA yield at the Belle kinematics [19] assuming that $D_{1}^{g}\left(z, M_{h} ; Q_{0}^{2}\right)=0$. We parametrize the error on the unconstrained $D_{1}^{g}$ by computing the denominator $D$ in Eq. (6) alternatively with $D_{1}^{g}\left(z, M_{h} ; Q_{0}^{2}\right)=D_{1}^{u}\left(z, M_{h} ; Q_{0}^{2}\right) / 4$ or $D_{1}^{u}\left(z, M_{h} ; Q_{0}^{2}\right)$. We have verified that these choices alter the $\chi^{2}$ of the $e^{+} e^{-}$fit in Ref. [19] by $10 \%-50 \%$, keeping always $\chi^{2} /$ d.o.f. $\lesssim 2$. The number $M$ of replicas is fixed by reproducing the mean and standard deviation of the original data points. For each option, it turns out that 200 replicas are sufficient. Hence, we have in total $M=600$ replicas.

For a total of 46 bins and 10 free parameters, we reach a global $\chi^{2} /$ d.o.f. $=2.08 \pm 0.09$. The SIDIS data contribute 

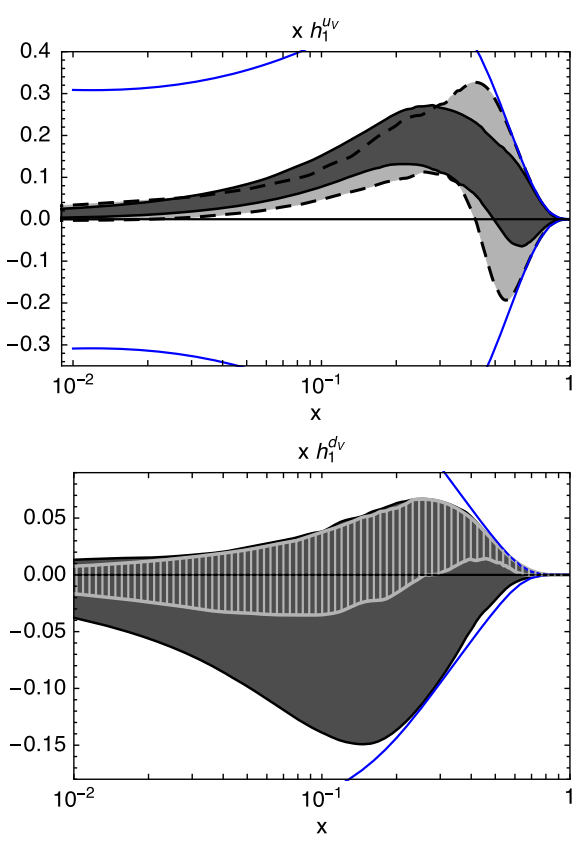

FIG. 1. The transversity $x h_{1}$ as a function of $x$ at $Q^{2}=2.4 \mathrm{GeV}^{2}$. Dark (blue) lines represent the Soffer bounds. Dark bands with solid borders for the global fit of this work including all options $D_{1}^{g}\left(Q_{0}^{2}\right)=0, D_{1}^{u}\left(Q_{0}^{2}\right) / 4$, and $D_{1}^{u}\left(Q_{0}^{2}\right)$. (Top) For valence up quark: comparison with our previous fit in Ref. [29] (lighter band with dashed borders). (Bottom) For valence down quark: comparison with this global fit with only $D_{1}^{g}\left(Q_{0}^{2}\right)=0$ (hatched area with lighter borders).

to the global $\chi^{2}$ by $\approx 38 \%$, most of which $(\approx 76 \%$ ) coming from COMPASS data points, due to their smaller errors. A significant amount $(\approx 40 \%)$ is contributed to the COMPASS $\chi^{2}$ budget by specific bins in the deuteron kinematics. The remaining $62 \%$ of the global $\chi^{2}$ comes from the STAR data and is dominated by the $\left|\boldsymbol{P}_{h T}\right|$ bins $(\approx 70 \%)$, while the $M_{h}$ bins contribute by $\approx 28 \%$ and the $\eta$ bins by a negligible $\approx 2 \%$.

In Fig. 1, the transversity $x h_{1}$ is displayed as a function of $x$ at $Q^{2}=2.4 \mathrm{GeV}^{2}$. The dark (blue) lines represent the Soffer bounds. The upper panel refers to the valence up component. Here, the lighter band with dashed borders corresponds to the $90 \%$ uncertainty band from our previous fit with only SIDIS and $e^{+} e^{-}$data [29]. The darker band with solid borders is the $90 \%$ uncertainty band from the new global fit discussed here, including all options $D_{1}^{g}\left(Q_{0}^{2}\right)=0, D_{1}^{g}\left(Q_{0}^{2}\right)=D_{1}^{u}\left(Q_{0}^{2}\right) / 4$, and $D_{1}^{g}\left(Q_{0}^{2}\right)=D_{1}^{u}\left(Q_{0}^{2}\right)$. However, the latter result is insensitive to the various choices for $D_{1}^{g}\left(Q_{0}^{2}\right)$. There is an evident gain in precision by including also the STAR data. The uncertainty of our previous fit in Ref. [29] (the lighter band) is comparable to the one obtained from the analysis of the Collins effect $[10,45]$. Hence, we deduce that the outcome of our global fit provides a substantial increase in the precision on $h_{1}^{u_{v}}$ and on the related tensor charge $\delta u$ with respect to all the other phenomenological extractions.
TABLE I. The tensor charge $\delta q$, truncated tensor charge $\delta \tilde{q}$, and isovector tensor charge $g_{T}$ at $90 \%$ confidence level (see text).

\begin{tabular}{lrrrr}
\hline \hline & \multicolumn{1}{c}{$\delta q$} & $\delta q$ & $\delta \tilde{q}$ & \multicolumn{1}{c}{$g_{T}$} \\
\cline { 2 - 5 }$\left(Q^{2}\left[\mathrm{GeV}^{2}\right]\right)$ & $Q_{0}^{2}=1$ & $Q^{2}=4$ & $Q^{2}=10$ & $Q^{2}=4$ \\
\hline $\mathrm{Up}$ & $0.43(11)$ & $0.39(10)$ & $0.32(8)$ & \multirow{2}{*}{$0.53(25)$} \\
Down & $-0.12(28)$ & $-0.11(26)$ & $-0.10(22)$ & \\
\hline \hline
\end{tabular}

The lower panel of Fig. 1 refers to the valence down component. Again, the darker band with solid borders corresponds to the $90 \%$ uncertainty band from the new global fit, including all options for $D_{1}^{g}\left(Q_{0}^{2}\right)$. The hatched area with lighter borders shows how the result is modified by including only the option $D_{1}^{g}\left(Q_{0}^{2}\right)=0$. At variance with the up quark, the valence down component $h_{1}^{d_{v}}$ is sensitive to the $D_{1}^{g}$ contribution to the cross section for $p-p$ collisions. Data on $\left(\pi^{+} \pi^{-}\right)$multiplicities in $p-p$ collisions would be very useful in constraining $D_{1}^{g}$. Finally, we notice that the unnatural behavior of $h_{1}^{d_{v}}$ at $x \gtrsim 0.1$ obtained in Ref. [29] has disappeared. The few COMPASS data points responsible for this anomalous trend [46] become statistically less relevant when including the STAR data, which in turn demonstrate their large impact on our knowledge of transversity.

In order to compare with other results, we have calculated at various scales the tensor charge $\delta q$ and the truncated tensor charge $\delta \tilde{q}$, which is obtained by restricting the integral in Eq. (9) to the experimental $x$ range [0.0065, $0.133]$. The values at $90 \%$ confidence level are listed in Table I. They are in very good agreement with other phenomenological extractions [10], and seem compatible with lattice simulations for $\delta d$, but not for $\delta u$ (see, e.g., Table IX in Ref. [47]). We have computed also the isovector tensor charge $g_{T} \equiv \delta u-\delta d$, whose systematic errors of lattice calculations are under better control. At $Q^{2}=4 \mathrm{GeV}^{2}$, our result is again in very good agreement with phenomenology (displaying a significantly smaller error with respect to our previous fit with only dihadron SIDIS and $e^{+} e^{-}$data [29]), but seems incompatible with most recent lattice simulations [10,47]. Lately, the authors of Ref. [48] have published a Monte Carlo reanalysis of the Collins effect supplemented by lattice input for $g_{T}$, showing that their result for $g_{T}$ is compatible with some lattice calculations, although their numerical values for $\delta u$ and $\delta d$ turn out to be largely incompatible. More work is needed along both lines of improving the precision of phenomenological extractions and of benchmarking lattice simulations, because a careful determination of $g_{T}$ is of paramount importance in detecting BSM effects [1], e.g., in neutron $\beta$ decay, where the experimental accuracy has now reached the $0.1 \%$ level [49].

In summary, we have presented an extraction of transversity at leading order in the strong coupling constant where, for the first time, we performed a global fit of all data for azimuthal asymmetries in the semi-inclusive 
production of charged pion pairs in deep-inelastic scattering, electron-positron annihilation, and proton-proton collisions. The uncertainty on the result for the valence up quark is smaller than any previous extraction. The large sensitivity of the valence down quark to the unconstrained gluon channel in dihadron fragmentation calls for data on pion pair multiplicities in proton-proton collisions, which are still missing. The calculated isovector tensor charge seems incompatible with most lattice calculations.

M. R. thanks U. D'Alesio and G. Bozzi for useful discussions. This work is supported by the European Research Council (ERC) under the European Union's Horizon 2020 research and innovation program (Grant No. 647981, 3DSPIN).

*marco.radici@pv.infn.it †alessandro.bacchetta@unipv.it

[1] A. Courtoy, S. Baessler, M. Gonzalez-Alonso, and S. Liuti, Phys. Rev. Lett. 115, 162001 (2015).

[2] T. Bhattacharya, V. Cirigliano, S. D. Cohen, A. Filipuzzi, M. Gonzalez-Alonso, M. L. Graesser, R. Gupta, and H.-W. Lin, Phys. Rev. D 85, 054512 (2012).

[3] D. Dubbers and M. G. Schmidt, Rev. Mod. Phys. 83, 1111 (2011).

[4] N. Yamanaka, B. K. Sahoo, N. Yoshinaga, T. Sato, K. Asahi, and B. P. Das, Eur. Phys. J. A 53, 54 (2017).

[5] J. Butterworth et al., J. Phys. G 43, 023001 (2016).

[6] A. Accardi et al., Eur. Phys. J. C 76, 471 (2016).

[7] R. L. Jaffe and X. Ji, Phys. Rev. Lett. 67, 552 (1991).

[8] M. Anselmino, M. Boglione, U. D’Alesio, A. Kotzinian, F. Murgia, A. Prokudin, and C. Türk, Phys. Rev. D 75, 054032 (2007).

[9] J. C. Collins, Nucl. Phys. B396, 161 (1993).

[10] Z.-B. Kang, A. Prokudin, P. Sun, and F. Yuan, Phys. Rev. D 93, 014009 (2016).

[11] T. C. Rogers and P. J. Mulders, Phys. Rev. D 81, 094006 (2010).

[12] R. L. Jaffe, X. Jin, and J. Tang, Phys. Rev. Lett. 80, 1166 (1998).

[13] J. C. Collins and G. A. Ladinsky, arXiv:hep-ph/9411444.

[14] A. Bianconi, S. Boffi, R. Jakob, and M. Radici, Phys. Rev. D 62, 034008 (2000).

[15] M. Radici, R. Jakob, and A. Bianconi, Phys. Rev. D 65, 074031 (2002).

[16] A. Bacchetta and M. Radici, Phys. Rev. D 67, 094002 (2003).

[17] D. Boer, R. Jakob, and M. Radici, Phys. Rev. D 67, 094003 (2003).

[18] A. Bacchetta, F. A. Ceccopieri, A. Mukherjee, and M. Radici, Phys. Rev. D 79, 034029 (2009).

[19] A. Courtoy, A. Bacchetta, M. Radici, and A. Bianconi, Phys. Rev. D 85, 114023 (2012).

[20] A. Bacchetta and M. Radici, Phys. Rev. D 70, 094032 (2004).

[21] M. Radici, A. M. Ricci, A. Bacchetta, and A. Mukherjee, Phys. Rev. D 94, 034012 (2016).
[22] A. Airapetian et al. (HERMES Collaboration), J. High Energy Phys. 06 (2008) 017.

[23] C. Adolph et al. (COMPASS Collaboration), Phys. Lett. 713B, 10 (2012).

[24] C. Adolph et al. (COMPASS Collaboration), Phys. Lett. 736B, 124 (2014).

[25] C. Braun (COMPASS Collaboration), EPJ Web Conf. 85, 02018 (2015).

[26] A. Vossen et al. (Belle Collaboration), Phys. Rev. Lett. 107, 072004 (2011).

[27] A. Bacchetta, A. Courtoy, and M. Radici, Phys. Rev. Lett. 107, 012001 (2011).

[28] A. Bacchetta, A. Courtoy, and M. Radici, J. High Energy Phys. 03 (2013) 119.

[29] M. Radici, A. Courtoy, A. Bacchetta, and M. Guagnelli, J. High Energy Phys. 05 (2015) 123.

[30] L. Adamczyk et al. (STAR Collaboration), Phys. Rev. Lett. 115, 242501 (2015).

[31] H. H. Matevosyan, A. Bacchetta, D. Boer, A. Courtoy, A. Kotzinian, M. Radici, and A. W. Thomas, Phys. Rev. D 97, 074019 (2018).

[32] X. Artru and J. C. Collins, Z. Phys. C 69, 277 (1996).

[33] R. Seidl et al. (Belle Collaboration), Phys. Rev. D 96, 032005 (2017).

[34] M. Stratmann and W. Vogelsang, Phys. Rev. D 64, 114007 (2001).

[35] See the Supplemental Material at http://link.aps.org/ supplemental/10.1103/PhysRevLett.120.192001 for details of the calculation of the matrix element for proton-proton collisions in Mellin space, for the fit to the Soffer bound at the starting scale, and for the formula of transversity evolved at $\mathrm{LO}$ in Mellin space.

[36] A. Bacchetta and M. Radici, Phys. Rev. D 74, 114007 (2006).

[37] W. Vogelsang, Phys. Rev. D 57, 1886 (1998).

[38] A. D. Martin, W. J. Stirling, R. S. Thorne, and G. Watt, Eur. Phys. J. C 63, 189 (2009).

[39] D. de Florian, R. Sassot, M. Stratmann, and W. Vogelsang, Phys. Rev. D 80, 034030 (2009).

[40] N. Sato, W. Melnitchouk, S. E. Kuhn, J. J. Ethier, and A. Accardi (Jefferson Lab Angular Momentum Collaboration), Phys. Rev. D 93, 074005 (2016).

[41] J. J. Ethier, N. Sato, and W. Melnitchouk, Phys. Rev. Lett. 119, 132001 (2017).

[42] A. Accardi and A. Bacchetta, Phys. Lett. 773B, 632 (2017).

[43] G. P. Salam and J. Rojo, Comput. Phys. Commun. 180, 120 (2009).

[44] F. A. Ceccopieri, M. Radici, and A. Bacchetta, Phys. Lett. 650B, 81 (2007).

[45] M. Anselmino, M. Boglione, U. D’Alesio, S. Melis, F. Murgia, and A. Prokudin, Phys. Rev. D 87, 094019 (2013).

[46] M. Radici, Proc. Sci., QCDEV2016 (2017) 013.

[47] T. Bhattacharya, V. Cirigliano, S. D. Cohen, R. Gupta, A. Joseph, H.-W. Lin, and B. Yoon (PNDME Collaboration), Phys. Rev. D 92, 094511 (2015).

[48] H.-W. Lin, W. Melnitchouk, A. Prokudin, N. Sato, and H. Shows, arXiv:1710.09858.

[49] V. Cirigliano, S. Gardner, and B. Holstein, Prog. Part. Nucl. Phys. 71, 93 (2013). 